\title{
深度光流估计方法研究进展
}

\author{
祖日然 ${ }^{1)}$, 包秀国 ${ }^{2)}$, 唐文忠 ${ }^{1)^{*}}$, 高科 ${ }^{3)}$, 张冬明 ${ }^{2)}$ \\ 1) (北京航空航天大学计算机学院 北京 100191) \\ ${ }^{2)}$ (国家计算机网络与信息安全管理中心 北京 100029) \\ 3) (中国科学院计算技术研究所智能信息处理重点实验室 北京 100190) \\ (tangwenzhong@buaa.edu.cn)
}

\begin{abstract}
摘 要: 结合深度学习模型实现光流端到端的计算是当前计算机视觉领域的一个研究热点. 文中对基于深度学习的 光流估计方法进行总结和梳理. 首先, 介绍了光流的起源与定义; 其次, 总结了现有的数据集合和评价指标; 最重要 的是, 着重从 3 个方面回顾了深度光流估计方法, 包括有监督的深度光流估计方法、无监督的深度光流估计方法以及 对现有光流估计方法的性能对比分析. 分析表明, 参照传统方法设计小而轻且泛化性能好的深度光流网络是未来的 研究方向. 在此基础上, 进一步分析和介绍了当下光流估计与视频分析任务联合学习的一系列代表性方法, 指出了 设计由任务驱动的深度光流网络是很有应用价值的研究方向. 最后, 总结了深度光流估计存在的问题和挑战, 并对 未来工作进行展望.
\end{abstract}

关键词: 光流; 光流估计; 深度学习; 视频分析

中图法分类号: TP391.41 DOI: 10.3724/SP.J.1089.2021.17931

\section{Research Progress of Deep Optical Flow Estimation}

\author{
Zu Yueran ${ }^{1)}$, Bao Xiuguo ${ }^{2)}$, Tang Wenzhong ${ }^{1)^{*}}$, Gao $\mathrm{Ke}^{3)}$, and Zhang Dongming ${ }^{2)}$ \\ 1) (School of Computer Science and Technology, Beihang University, Beijing 100191) \\ ${ }^{2)}$ (The National Computer Network Emergency Response Technical Team Coordination Center of China, Beijing 100029) \\ ${ }^{3)}$ (Key Laboratory of Intelligent Information Processing, Institute of Computing Technology, Chinese Academy of Sciences, Beijing 100190)
}

\begin{abstract}
Combining the deep learning model to compute end-to-end optical flow is a hot topic in current computer vision field. The optical flow estimation methods based on deep learning are summarized and reviewed. Firstly, the origin and concept of optical flow is introduced. Secondly, the optical flow datasets and evaluation metrics are summarized. Most importantly, classical methods are introduced and the deep optical flow estimation methods are reviewed in three aspects, including supervised deep optical flow estimation methods, unsupervised deep optical flow estimation methods and the performance of these methods. The analysis shows designing compact and generalized deep optical flow model is the future research direction. On this basis, the joint learning of optical flow estimation and specific video analysis tasks are introduced. It is pointed out that the design of task-driven deep optical flow network is a valuable research direction in practice application. Finally, the problems and challenges of deep optical flow estimation are summarized and the future work of this field is prospected.
\end{abstract}

收稿日期：2019-07-02; 修回日期：2019-07-15. 基金项目：国家自然科学基金(51475025); 国家重点研发计划(2016YFB0801203); 北京市科学技术委员会基金(Z171100000117010). 祖曰然(1988一), 女, 博士研究生, CCF 学生会员, 主要研究方向为计算机视觉; 包秀国(1963-), 男, 博士, 教授级高级工程师, 主要研究方向为计算机网络安全; 唐文忠(1967一), 男, 博士, 研究员, 博士生导师, 论文通讯作者, 主要研究方向为大规模信息化工程、工业互联网; 高科(1982一), 女, 博士, 副研究员, CCF 会员, 主要研究方向为多 媒体内容分析和检索技术; 张冬明(1977一), 男, 博士, 研究员, 硕士生导师, CCF 会员, 主要研究方向为视频编码、多媒体内容检索. 
Key words: optical flow; optical flow estimation; deep learning; video analysis

光流估计是计算机视觉的基本任务之一. 自 从 1981 年提出变分求解方法发展至今, 光流估计 仍是一个没有解决的问题. 近年来, 光流估计在各 方面都取得了快速的发展，因其应用的广泛性，使 之成为一个研究热点. 光流在工业和军事应用领 域具有重要的现实意义 ${ }^{[1]}$, 在诸如完成各种工业或 军事任务的机器人视觉系统、地对空导弹火控系 统、自动飞行器着陆与精确导航系统、空间卫星跟 踪系统、战场的动态分析系统、医学图像分析中器 官异常分析和诊断系统，以及进行资源勘探、天气 预报、军事侦察的航空或卫星照片的自动分析系统 等中均有应用. 本文只论述光流在视频分析领域 的应用，如动作识别和视频分割等方面.

本文从光流的起源和定义人手, 介绍了光流 的数据集和评价指标; 随后, 介绍了光流估计的经 典方法并着重回顾了基于深度学习的方法; 在此 基础上, 进一步介绍了光流估计与视频分析任务 联合学习的方法; 最后, 总结深度光流估计存在的 问题和挑战，并对未来工作进行展望.

\section{1 光流的起源和定义}

光流的起源可以追溯到 1951 年, Prentice 等 ${ }^{[2]}$ 是 20 世纪最有影响力的心理学家之一, 通过在日 常生活中观察世界物体的运动规律, 对认知运动 产生了兴趣, 提出了生态心理学, 在没有附加的认 知建立或处理的情况下，通过意识直接感知环境， 为后续光流的定义和研究奠定了基础.

“光流”2 字看上去比较抽象, 可以这样理解, 当人的眼睛观察运动物体时, 物体的景象在人眼 的视网膜形成一系列连续变化的图像, 这一系列 连续变化的信息不断“流过”视网膜(即图像平面), 好像一种光的“流”故称之为光流.

光流被定义为一种透明的运动，是观察者和 场景之间的相对运动. 光流是基于像素点定义的, 所有光流的集合称为光流场. 通过对光流场进行 分析, 可以得到物体相对观察者的运动场. 光流估 计就是计算光流的一系列方法.

通俗地讲, 就是经过一个图像序列把每幅图像 中每个像素的运动速度和运动方向计算出来, 即得 到了光流场. 第 $t-1$ 帧时 $A$ 点的位置为 $\left(x_{1}, y_{1}\right)$, 那 么在第 $t$ 帧时再找到 $A$ 点, 假如它的位置为 $\left(x_{2}, y_{2}\right)$, 就可以确定 $A$ 点的运动 $\left(u_{x}, u_{y}\right)=\left(x_{2}, y_{2}\right)-\left(x_{1}, y_{1}\right)$.
向量 $\left(u_{x}, u_{y}\right)$ 就是 $A$ 点的光流, 其包含 $x$ 方向和 $y$ 方向的运动, 光流的值是亚像素的浮点数值. 如图 1 所示, 左图中的 $A$ 点运动到右图, 箭头表示的是 $A$ 点的光流.

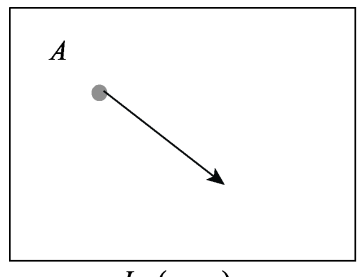

$I_{t-1}\left(x_{1}, y_{1}\right)$

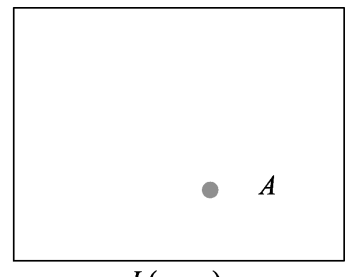

$I_{t}\left(x_{2}, y_{2}\right)$
图 1 单个像素点的光流示意图

\section{2 光流的数据库和评价指标}

近年来, 随着光流的发展, 其公开的数据库也 在不断更新. 本文介绍 2011-2019 年发布的光流 相关数据集合.

2011 年由 Baker 等 ${ }^{[3]}$ 提出的公开测评数据集, 图像数量较少, 大部分都是合成的图像, 有几对现 实图像, 包含 8 对训练集. 数据集位移较小, 位移 小于 1 个像素.

卡尔斯鲁厄理工学院技术学院丰田研究院芝加 哥分院(Karlsruhe Institute of Technology and Toyota Technological Institute, KITTI) 在 2012 年和 2015 年分 别提出了 2 个数据库 KITTI $2012^{[4]}$ 和 KITTI $2015^{[5]}$, 主要是针对无人驾驶, 利用行车记录仪器和多个车 载传感器拍摄得到的街景图像, 其中图像包含的 物体有限(如车、人、路等). 光流估计是其中的一 个基本任务, 数据集的标签不是逐像素的, 是采样 得到的半稠密光流. 图像的分辨率为 $1242 \times 376$ 像 素. KITTI2015 中的数据是彩色图, 且包含的运动 更复杂.

在 2012 年 ECCV 会议上, 德国马克斯·普朗克 研究所(Max Planck Institute), 简称马普所, 提出 了一个 MPI Sintel ${ }^{[6]}$ 数据集, 包含 1041 对图像帧. Sintel 数据集来源于一个全部开源的 3D 动画电影, 讲述的是主人公 Sintel 与一条龙的故事, 共 $15 \mathrm{~min}$. 此数据集含有 2 个版本: clean 版本和 final 版本. clean 版本包含的是只有光照变换的图像, 而 final 版本包含了模糊、动作等与现实视频相当的特效, 动作比较复杂. 为了表现其与现实视频的相似度, 原文作者将其与真实图像进行了对比统计. 
2015 年, 德国弗莱堡大学的 Brox 团队提出了 FlyingChairs 数据集 ${ }^{[7]}$, 数量达 22872 幅. 图像是 合成的，其中背景来自网络相册 Flickr，椅子是后 来加上去的 $3 \mathrm{D}$ 模型. 2016 年又提出了适合应用卷 积神经网络 (convolutional neural networks, CNN)的 最大数据集合, 包含 FlyingThings3D, Monkaa, Driving ${ }^{[8]} 3$ 种不同场景下的合成图像，共 64000 幅.

由于目前没有直接检测到光流的传感器，如 果用人工标注每个像素，非常耗时耗力. 虽然现有 合成的数据库能在一定程度上反映真实数据, 但 是与真实视频的光流存在着差距. 如何得到真实 视频的光流是一个比较难解决的问题. 随着硬件 设备的发展，2017 年称为 Slow Flow ${ }^{[9]}$ 的数据集利 用快速相机记录下每个像素的运动，其包含 293 对 图像帧, 并包含 3 个速度下的图像. 该数据集不足 之处是其仍然受限于相机设备, 不能满足处理现 实世界视频的需求.

目前光流估计方法的流行评测数据集是 Middlebury, MPI Sintel 和 KITTI, 其他的大数据集 一般用于深度 $\mathrm{CNN}$ 的训练. 几个光流数据库的比 较如表 1 所示.

表 1 光流数据集对比

\begin{tabular}{|c|c|c|c|c|}
\hline 数据集 & 帧对数 & 年份 & 发布机构 & 特点 \\
\hline Middlebury $^{[3]}$ & 72 & 2007 & Middleb & 位移小 \\
\hline KITTI2012 $2^{[4]}$ & 194 & 2012 & KITTI & 光流稀疏 \\
\hline KITTI2015 $5^{[5]}$ & 200 & 2015 & 121111 & 光流稀疏 \\
\hline MPI Sintel ${ }^{[6]}$ & 1041 & 2012 & MPI & 位移大 \\
\hline FlyingChairs $^{[7]}$ & 22872 & 2015 & Freiburg University & 合成图像 \\
\hline FlyingThings $3 \mathrm{D}^{[8]}$ & 21818 & 2016 & Freiburg University & 合成图像 \\
\hline Monkaa $^{[8]}$ & 8591 & 2016 & Freiburg & 合成图像 \\
\hline Driving $^{[8]}$ & 4392 & 2016 & Freiburg University & 合成图像 \\
\hline Slow Flow ${ }^{[9]}$ & $296 \times 3$ & 2017 & MPI & 真实图像 \\
\hline
\end{tabular}

光流的通用评价指标有 2 种，一种是端点到端 点误差(end point error, EPE), 如

$$
\mathrm{EPE}=\sqrt{\left(u-u_{\mathrm{GT}}\right)^{2}+\left(v-v_{\mathrm{GT}}\right)^{2}}
$$

其中, $(u, v)$ 表示光流在 $x$ 方向和 $y$ 方向的位移大小; $\left(u_{\mathrm{GT}}, v_{\mathrm{GT}}\right)$ 表示光流的标签值, GT 是 Ground Truth 的简写.

另一个评价指标是平均角度误差(angular error, $\mathrm{AE})$, 如

$$
\mathrm{AE}=\cos ^{-1}\left(\frac{1.0+u \times u_{\mathrm{GT}}+v \times v_{\mathrm{GT}}}{\sqrt{1.0+u^{2}+v^{2}} \sqrt{1.0+u_{\mathrm{GT}}^{2}+v_{\mathrm{GT}}^{2}}}\right)
$$

这 2 个评价指标的结果是正相关的，最常用的 是整个数据集合中所有图像的平均 EPE(average
EPE, AEPE)

除了传统的评价指标, KITTI 和 MPI Sintel 数 据集上都有自己的特殊评价指标，如表 2 所示. KITTI 数据集合区分前景和背景, 定义了超出一定 范围(如大于 3 个像素)的光流为异常光流 $F 1$, 包含 在整幅图上的异常光流的百分比 Fl-all、在背景上 的平均百分比 Fl-bg、前景上的百分比 Fl-fg 等. 在 MPI Sintel 数据集中还细化了评价指标, 包括 2 帧 中同时出现/同时未出现的像素, 根据距离遮挡边 界的距离大小进行分类，根据不同光流速度的区 域进行划分的评价标准.

\begin{tabular}{|c|c|c|}
\hline 数据集 & 评价指标 & 解释 \\
\hline \multirow{4}{*}{ KITTI } & $\mathrm{Fl}$ & 异常光流占的百分比 \\
\hline & bg & 背景区域异常光流占的百分比 \\
\hline & fg & 前景区域异常光流占的百分比 \\
\hline & all & 异常光流占所有标签的百分比 \\
\hline \multirow{9}{*}{ MPI Sintel } & EPE & 整幅图像上的端点误差 \\
\hline & $\begin{array}{l}\text { EPE } \\
\text { matched }\end{array}$ & 相邻帧中可见区域的端点误差 \\
\hline & $\begin{array}{l}\text { EPE } \\
\text { unmatched }\end{array}$ & 相邻帧中不可见区域的端点误差 \\
\hline & $\mathrm{d} 0-10$ & $\begin{array}{l}\text { 距离最近遮挡边缘在 } 0 \sim 10 \text { 像素的端点 } \\
\text { 误差 }\end{array}$ \\
\hline & $\mathrm{d} 10-60$ & $\begin{array}{l}\text { 距离最近遮挡边缘在 } 10 \sim 60 \text { 像素的端 } \\
\text { 点误差 }\end{array}$ \\
\hline & d60-140 & $\begin{array}{l}\text { 距离最近遮挡边缘在 60 140 像素的端 } \\
\text { 点误差 }\end{array}$ \\
\hline & s0-10 & 速度在 $0 \sim 10$ 像素的帧区域端点误差 \\
\hline & s10-40 & 速度在 10 40 像素的帧区域端点误差 \\
\hline & s $40+$ & 速度在 40 以上像素的帧区域端点误差 \\
\hline
\end{tabular}

表 2 不同数据集下的评价指标

\section{3 光流估计的方法}

光流估计方法的发展和关系如图 2 所示. 按照 时间顺序大致分为 3 类方法: 基于能量函数的方 法、由稀疏到稠密的方法和基于深度学习的光流估 计方法.

在 1981-2016 年期间, 最小化能量函数方法 是求光流的主要方法，此类方法是经典的求光流 的方法, 缺点是计算复杂度较高, 且容易陷人局部 最优值. 自 Brox 等 ${ }^{[10]}$ 在能量函数中插人了匹配项, 在 2011-2017 年出现了很多先求匹配值作为光流 的初始化再插值的优化方法. 此类方法获得更优 的光流初始值, 鲁棒的匹配和插值结果有助于最 后能量函数的优化. 2015 年, FlowNet ${ }^{[7]}$ 首次提出, 将光流在能量函数上的在线优化过程转为在 $\mathrm{CNN}$ 
上进行的离线优化过程. 为了进一步提高深度光 流估计的精度和运算速度, 基于能量函数的有效 策略被用到网络结构的设计中. 在无监督的方法 中, 损失函数的设计与能量函数方程是对应的. 3 类 方法的内在联系如图 2 所示, 基于能量函数方程中 的数据项对应匹配方法和深度网络结构中的编码 部分，包含提取特征和计算 2 幅图像的相关性; 而 平滑项对应插值方法和网络结构中的解码部分, 用解卷积实现.

本文简单介绍了 Horn-Schunck (HS) 和金字塔 Lucas-Kanade (LK) 2 个经典的光流估计方法, 重点 介绍基于深度学习的光流估计方法. 这 2 个经典的 方法与深度光流估计的方法联系最紧密, 其中 HS 方法与损失函数相关，金字塔 LK 方法有助于网络 结构的设计.

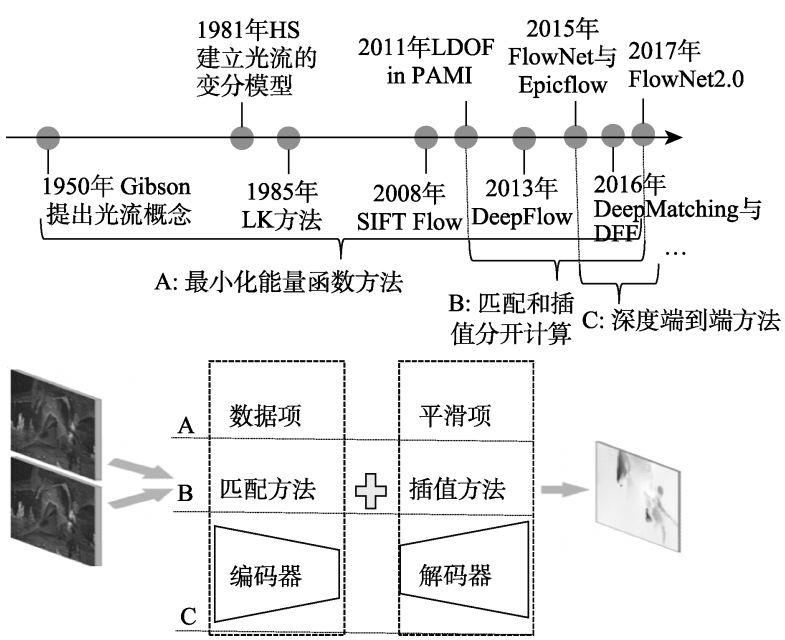

图 2 光流估计方法发展及关系图

\section{1 经典方法}

传统方法始于 1981 年 Horn 等 ${ }^{[11]}$ 提出的 HS 方 法, 其基于 2 个假设：相邻帧之间的像素值不变和 相邻 2 帧的位移很小. 用一个能量方程来表示目标 函数, 求其最优值从而估计出最终光流值. 根据所 求光流区域的范围, 分为全局方法和局部方法. 本 文只介绍 2 个经典的方法, 关于传统方法的详细综 述见参考文献[12-13].

\subsubsection{HS 方法}

$\mathrm{HS}^{[11]}$ 方法基于 2 点假设.

假设 1. 灰度值守恒假设. 假设第 1 帧到第 2 帧图像的灰度值没有发生变化, 即

$$
I(x, y, t)=I(x+u, y+v, t+1)
$$

假设 2. 光流的全局平滑. $u$ 和 $v$ 随着像素点移 动而发生的改变是缓慢的，局部区域速度的空间 变化率为 0 , 即

$$
\ell_{\mathrm{c}}^{2}=\left(\frac{\partial u}{\partial x}\right)^{2}+\left(\frac{\partial u}{\partial y}\right)^{2}+\left(\frac{\partial v}{\partial y}\right)^{2}+\left(\frac{\partial v}{\partial y}\right)^{2}
$$

结合 2 个约束条件, 可以建立极小化方程

$$
\ell^{2}=\iint \alpha^{2} \ell_{\mathrm{c}}^{2}+\left(I_{x} u+I_{y} v+I_{t}\right)^{2} \mathrm{~d} x \mathrm{~d} y
$$

用迭代的方式求解极小化方程, 其最优解为 光流的值. 此类方法的优点是可以求出光流的值, 缺点是对噪声敏感, 只能处理小位移. 后来有很多 学者通过修改或添加约束 ${ }^{[14-16]}$ 来改进式(5).

\section{1 .2 金字塔 LK 方法}

$L K$ 方法 ${ }^{[17]}$ 针对稀疏点估计光流, 所以又称为 稀疏光流法. 分析某像素点附近 $n \times n$ 区域, 则可以 建立类似 $n \times n$ 个方程.

$$
\left[\begin{array}{ll}
I_{x_{i}} & I_{y_{i}}
\end{array}\right]\left[\begin{array}{l}
u \\
v
\end{array}\right]=-\left[I_{t_{i}}\right], i=1,2, \cdots, n
$$

这个问题就变成了一个超定问题，可以用最小 二乘法来解. 式(6)可以写成式(7) 式(9)进行求解.

$$
\begin{gathered}
\boldsymbol{A u}=\boldsymbol{b} \\
\boldsymbol{b}=\left(\boldsymbol{A}^{\mathrm{T}} \boldsymbol{A}\right)^{-1} \boldsymbol{A}^{\mathrm{T}} \boldsymbol{b} \\
\boldsymbol{A}^{\mathrm{T}} \boldsymbol{A}=\left[\begin{array}{cc}
\sum I_{x}^{2} & \sum I_{x} I_{y} \\
\sum I_{x} I_{y} & \sum I_{y}^{2}
\end{array}\right]
\end{gathered}
$$

金字塔 LK 方法 ${ }^{[18]}$ 在每一层图像中用 LK 方法 计算当前分辨率下的光流, 然后把结果乘以尺度 系数作为下一层光流的初始化. 在计算下一层的 光流时, 通过上采样和变形方法得到第 1 帧的近似 帧, 然后求取剩余的光流值, 减小了计算量. 为了 更好地求大位移的光流，引人了金字塔，最小分辨 率的金字塔能得到最小的位移，最下层的金字塔 就可以得到更大的位移，如图 3 所示.

此方法的优点是可以求得大位移的光流, 缺 点是对小物体大位移的光流易丢失，导致对精细

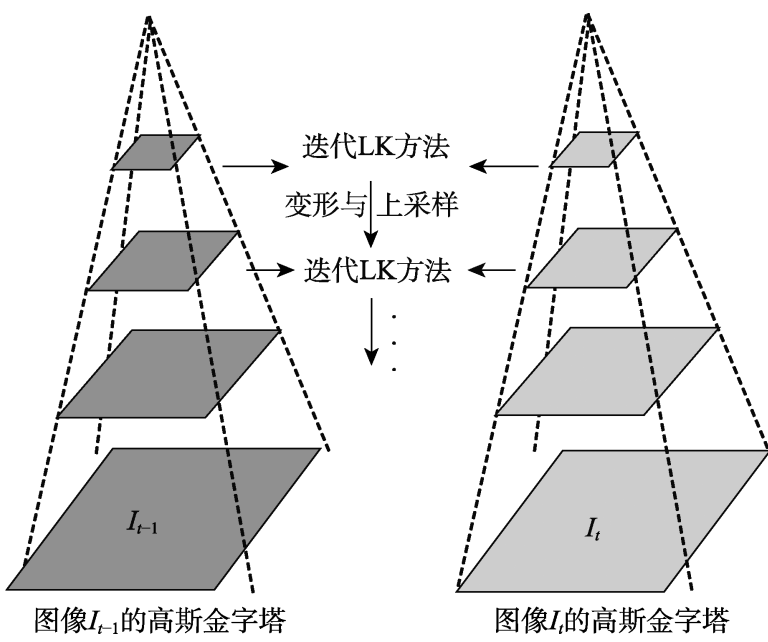

图 3 金字塔 LK 方法示意图 ${ }^{[18]}$ 
结构的过度平滑. 金字塔的策略也可以用在 HS 方 法中.

\section{2 深度光流估计方法}

2012 年以来, 深度学习在计算机视觉领域得到 广泛的应用且取得了较好的效果. 深度 CNN 依赖于 大量的数据集合, 由于难以获得光流的标注, 数据 集合的数量受限. 用深度学习的方法直接学习光流 始于 2015 年计算机视觉会议中提出的 FlowNet $1.0^{[7]}$, 接着出现了 SpyNet ${ }^{[19]}$, FlowNet2. $0^{[20]}$, 以及最新的 PWC-Net ${ }^{[21]}$, LiteFlowNet ${ }^{[22]}$ 和 IRRNet ${ }^{[23]}$, 还有一些 非监督的深度光流估计方法. 下面分别对其进行介 绍和分析.

\subsection{1 有监督的深度光流估计方法}

初次尝试用端到端的 CNN 进行光流估计的是 2015 年德国弗莱堡大学的 Dosovitskiy 等 ${ }^{[7]}$ 提出的 FlowNet1.0. 其 2 种网络结构图, 如图 4 所示.

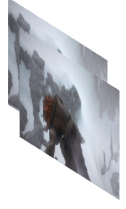

输人

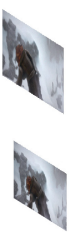

输人
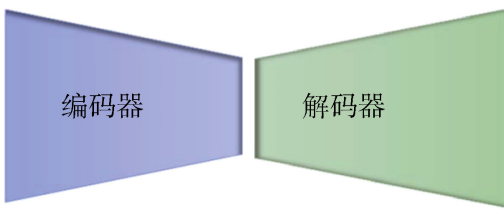

FlowNetS

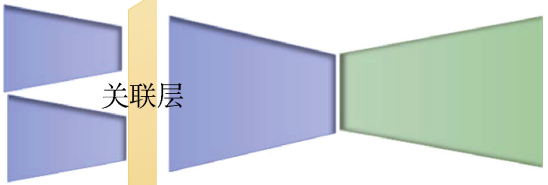

FlowNetC

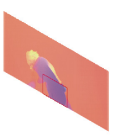

输出

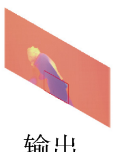

输出
图 4 FlowNet1.0 的 2 种网络结构示意图 ${ }^{[8]}$

一种是简单的光流网络(FlowNetS), 其输人为 相邻 2 帧, 先通过卷积神经网络下采样得到图像的 特征向量, 然后为了得到逐像素的光流, 在后面网 络用反卷积的方法得到逐像素的光流结果; 另一 种为关联光流网络 (FlowNetC), 受传统方法 ${ }^{[10]}$ 在 光流估计方法中加人匹配项的启发，其计算 2 幅图 像的匹配后，通过特征空间求光流. 具体做法是先 分别求 2 幅输人图像特征, 再加人一个关联层, 相 当于计算 2 幅输人图像的近似匹配程度后, 再继续 提取其他图像的特征.

具体的训练数据集合来自其发布的一个带有 20000 多张图片的人工合成数据集合 FlyingChiars. 其背景来自 Flickr, 前景是具有 3D 信息的椅子. FlowNet1.0 这个网络可以求出光流，但是其准确 率不高, 尤其是背景部分出现很多错误, 精度没有 传统方法高.
2017 年，马普所的 Black 团队提出了一种将经 典的空间金字塔的方法与深度学习结合的方法, 简称 $\mathrm{SpyNet}^{[19]}$. 其基本思想是: 为减轻光流网络 的负担，位移大的光流通过金字塔结构实现. 参照 传统的金字塔 $\mathrm{LK}^{[17]}$ 方法使用了由粗到细的空间金 字塔网络, FlowNet1.0 的问题是, 将 2 张图叠加放 到 CNN 中, 当 2 帧图像之间的运动较大时, 时空 卷积核会失效. 也就是说, 如果一个卷积核窗口与 另一帧的像素不重叠，那么此卷积核就没有得到 有效的信息. 光流的计算包括 2 个关键问题：(1) 长期依赖的大位移光流; (2) 细节的和亚像素的精 确运动边界. FlowNet1.0 同时解决这 2 个问题, 而 SpyNet 试图减轻 FlowNet 对于问题(1)的负担.

SpyNet 按照金字塔下采样, 先从最小分辨率 的图像开始计算, 得到的结果作为下一层的输人. 此方法的优点是减少了参数量, 比 FlowNet1.0 的 参数量少 $96 \%$. 其中每一层网络的位移都很小, 便 于神经网络学习, 学习到的时空卷积核与传统的 时空滤波器很像. 其缺点是精度一般, 且每一层网 络都需要单独训练.

针对 FlowNet1.0 精度较差的问题, 德国弗莱 堡大学团队在 2017 年又提出了 FlowNet2. $0^{[20]}$, 其 精度得到大幅度提高, 可以达到与传统方法差不 多的精度. 主要在 3 个方面进行了改进: (1) 在网 络结构上引用了迭代的网络结构; (2) 在训练顺序 上, 发现先在较简单的数据集合上进行训练, 然后 用 $3 \mathrm{D}$ 数据集(Things3D)进行微调, 可以提高精度. 这在一定程度上表明, 深度学习网络需要一点点 地学，遵循先易后难的原则; (3) 为了进一步提高 小位移光流的精度, 加人了一个小位移的数据集 合, 专门设计了训练小位移光流的网络, 通过减少 卷积核的步数来减小感受野. 其网络结构如图 5 所 示. 此方法的优点是精度大幅提高; 缺点是网络复 杂, 训练参数量大, 占用内存大, 较难训练.
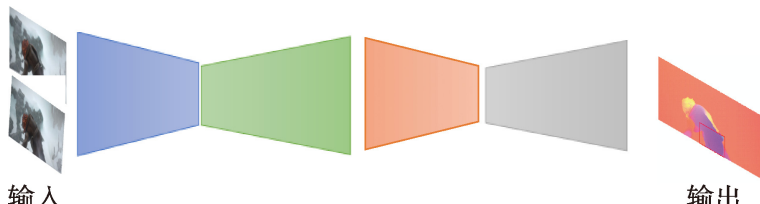

图 5 FlowNet2.0 网络结构示意图 ${ }^{[20]}$

Sun 等 ${ }^{[21]}$ 在 2018 年提出一个既简洁又有效的 CNN 模型 PWC-Net，并在 MPI Sintel 和 KITTI2015 数据集上达到了很高的精度, 其网络结构如图 6 所示. 


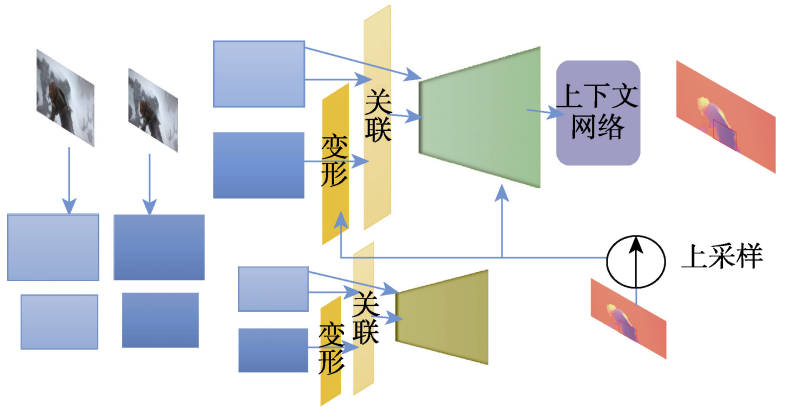

图 6 PWC-Net 网络结构示意图 ${ }^{[21]}$

PWC-Net 结合了在光流估计中常用的设计规 则: 金字塔(pyramid)、扭曲 (warping)和代价体 (cost volume). 把光流的求解空间映射到一个可学习的 特征空间金字塔, 取代 SpyNet 中的图像金字塔, 并用扭曲的方法估计出第 2 帧的特征，用得到的 2 帧特征建立代价体; 然后用 $\mathrm{CNN}$ 进行解码光流的 解. 因为代价体是比原像素本身更好的特征表达, 所以一个简洁的解码网络就可以得到光流的解. 此方法应用了最先进的深度网络结构, 其中在光 流估计的网络中加人了 DenseNet ${ }^{[24]}$ 的设计思想, 并且在上下文网络中使用了膨胀卷积核网络 ${ }^{[25]}$. 此方法的精度比 FlowNet2.0 高, 而且参数量是 FlowNet 2.0 的 1/17, 速度快 2 倍. 与此方法类似的 一个方法是 LiteFlowNet ${ }^{[22]}$, 其通过在每一个金字 塔层添加一个串联网络, 使前向传播预测光流更 为有效, 并添加了一个光流归一化层来改善异常 值和模糊边界的情况. 这个层是通过使用特征驱 动的局部卷积来实现的，也采用了一个有效的金 字塔特征提取结构，并采用特征变形，而不是像 FlowNet2.0 中所做的图像变形.

IRRNet ${ }^{[23]}$ 是 PWC-Net 的改进, 充分利用迭代 的思想，与 FlowNet2.0 的区别是把迭代放在网络 结构内部, 并实现了参数共享, 其参数量减少且精 度得到提高. 网络结构如图 7 所示. 另外, H3D $\mathrm{D}^{[26]}$ 方法是把金字塔用到了离散匹配的概率分布上， 同时可以计算匹配结果的信任度. 此外，还有一些

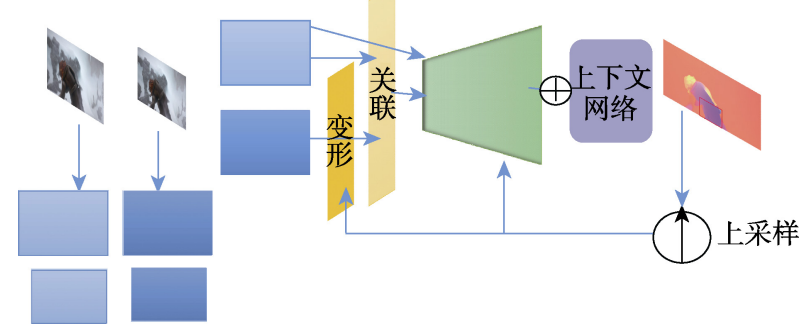

图 7 IRRNet 网络结构示意图

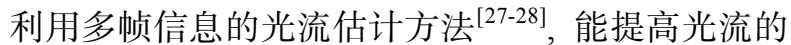
精度, 但是网络结构较 2 帧网络复杂一些.

综上，有监督的 FlowNet 的优点是在测试阶段 速度快, 缺点是其依赖于数据集合的数量、普适性差 且需要硬件支持. 因此, 设计出参数量更少的网络 结构, 更好地与传统方法相结合是未来的研究方向.

\subsection{2 无监督的深度光流网络}

因为光流的标签难以获得, 而且传统的方法 一直是使用非监督的方法求解最优化问题; 深度 神经网络本身就是一种优化方法，所以用非监督 的方法理论上可以对光流进行估计.

$\mathrm{Yu}$ 等 ${ }^{[29]}$ 提出用一个非监督的方法训练网络, 其代价方程是基于传统的光流约束条件：亮度约 束和平滑约束. 其损失函数是可微的，可以用反向 传播的方法传给网络的前一层进行无监督的训练. 该方法采用全卷积神经网络 (fully convolutional neural networks, FCNN), 其网络结构是 FlowNetS, 损失函数仍然使用数据项和平滑项的约束，中间 加了一层空间转换层来完成变形; 其效果仍然没 有监督的方法好. 最新的方法有 SelFlow ${ }^{[30]}$, 它充 分利用多帧信息, 并利用了额外的数据集合进行 学习, 使精度得到很大提高.

监督方法需要大量的标签数据, 现有的方法 用的都是合成的、非真实的数据集合; 而无监督的 方法可以利用现实生活中的视频进行训练, 但是 其损失函数依赖于基本的假设, 如亮度一致性和 局部平滑一致性. 这些假设对运动边界上的光流 并不适用，文献[31]提出一种用对抗神经网络进行 半监督学习的方法, 网络中并不是直接用显式假 设，而是用对抗损失获取光流变形误差图的结构 模式. 这种设计使假设是学习出来的, 而不是人工 设计的假设损失函数, 运算结果的精度介于监督 和无监督的方法之间.

无监督深度网络光流估计方法的优点是无须 标注数据就可以估计光流, 缺点是精度低于有监 督的方法. 因此, 设计更好的网络结构, 提高网络 精度是一个有价值的研究方向. 综上所述, 传统的 能量函数方程如何指导深度光流网络的损失函数, 以及进一步提高无监督深度光流估计网络的精度, 是未来的研究方向.

\subsection{3 光流估计方法的结果比较与分析}

在数据集合 KITTI 和 MPI Sintel 上将深度光流 网络的结果进行比较和分析, 总结现有深度光流 网络存在的问题和挑战.

表 3 所示为当下光流估计的前沿算法, 包含 7 
个传统方法 ${ }^{[32-38]}$ 和 6 个深度光流方法: FlowNet1. $0^{[7]}$, SpyNet ${ }^{[19]}$, FlowNet2.0 $0^{[20]}$, PWC-Net ${ }^{[21]}$ 及其变形, LiteFlowNet $^{[22]}$ 与 IRRNet ${ }^{[23]}$, 分别在 MPI Sintel 和 KITTI 数据集合上的测试结果的汇总. 其中 $\mathrm{ft}$ 表示 微调 finetune 的缩写, 评价指标为 AEPE.

通过分析表 3 可知, FlowNet2.0, PWC-Net 和 IRRNet 的性能达到甚至超过了传统方法. 另外 2 种网络的效果一般, 没有传统方法好. 另一个现象 是, 每个网络都需要微调, 在各自的数据集合上微 调才能得出较好的效果, 微调后的效果通常会好 很多. 例如, PWC-Net 在 KITTI2015 上微调之前的 $\mathrm{AEPE}=10.35$, 而微调之后 $\mathrm{AEPE}=2.16$, 相差约 5
倍. 在 Sintel_Final 的训练集上的微调后的结果为 2.08 , 而微调前的结果为 3.93 , 相差约 2 倍. 这说 明深度光流网络的泛化性能比较差. 遇到新的数 据集合需要重新对网络进行微调训练，才能得到 较好的结果.

表 4 是对几个深度光流网络的参数、内存和训 练时间的汇总. 由参数量可知, SpyNet 和 PWC-Net 的参数量较少, 且占用内存也较少. 但是训练的时 间仍然很长，需要 $4 \sim 5$ 天的时间. 前向传播和后向 传播都需要比较长的时间. 因此, 如何设计出参数 量小的网络，以及如何缩短训练时间是下一步的 研究方向.

表 3 各种方法在 Sintel 和 KITTI 数据集合上的结果汇总

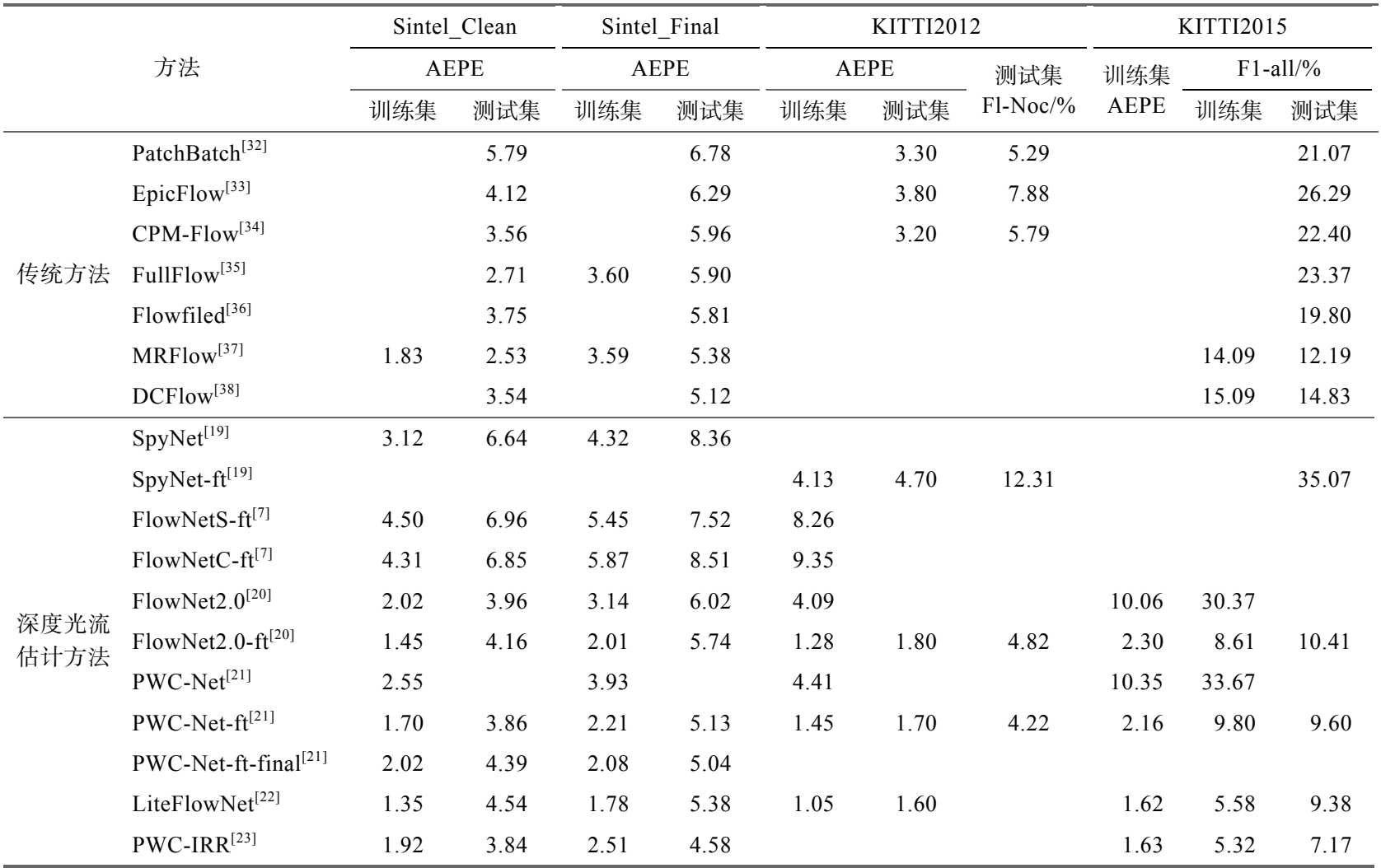

注. Fl-Noc 表示异常光流占所有非遮挡像素的百分比; F1-all 表示异常光流占所有标签的百分比.

表 4 各个网络的参数、内存和训练时间对比

\begin{tabular}{|c|c|c|c|c|c|c|c|}
\hline \multirow{2}{*}{ 网络 } & \multirow{2}{*}{$10^{-6} \times$ 参数量 } & \multirow{2}{*}{ 参数比 $/ \%$} & \multirow{2}{*}{ 内存/MB } & \multirow{2}{*}{ 内存占比 $/ \%$} & \multirow{2}{*}{ 训练天数/天 } & \multicolumn{2}{|c|}{ 传播时间 $/ \mathrm{ms}$} \\
\hline & & & & & & 前向 & 后向 \\
\hline FlowNetS $^{[7]}$ & 38.60 & 23.80 & 154.5 & 24.20 & 4 & 11.40 & 16.71 \\
\hline FlowNetC ${ }^{[7]}$ & 39.17 & 24.11 & 156.4 & 24.49 & 6 & 21.69 & 48.67 \\
\hline FlowNet2. $0^{[20]}$ & 162.49 & 100.00 & 638.5 & 100.00 & $>14$ & 84.80 & 78.96 \\
\hline SpyNet ${ }^{[19]}$ & 1.20 & 0.74 & 9.7 & 1.52 & & & \\
\hline PWC-Net ${ }^{[21]}$ & 8.75 & 5.38 & 41.1 & 6.44 & 4.8 & 28.56 & 44.37 \\
\hline PWC-Net-small ${ }^{[21]}$ & 4.08 & 2.51 & 22.9 & 3.59 & 4.1 & 20.76 & 28.44 \\
\hline
\end{tabular}


图 8 所示为各光流估计方法的时间-精度关系 图，其中 PWC-Net 达到了很好的平衡. 可以看出, 深度光流网络的测试时间很短，可以达到实时；而 传统方法目前在 CPU 上可以达到秒级. 因此, 设 计出实时地在 CPU 上进行光流估计的方法是一个 挑战.

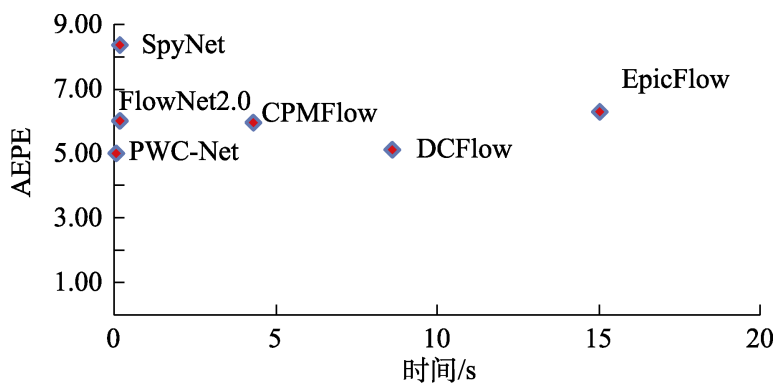

图 8 各光流估计方法的时间-精度关系图

图 9 所示为各光流估计方法的参数量-精度关 系图. 通过对比发现，如何设计参数量更小且精度 更高的深度光流估计网络也是一个挑战.

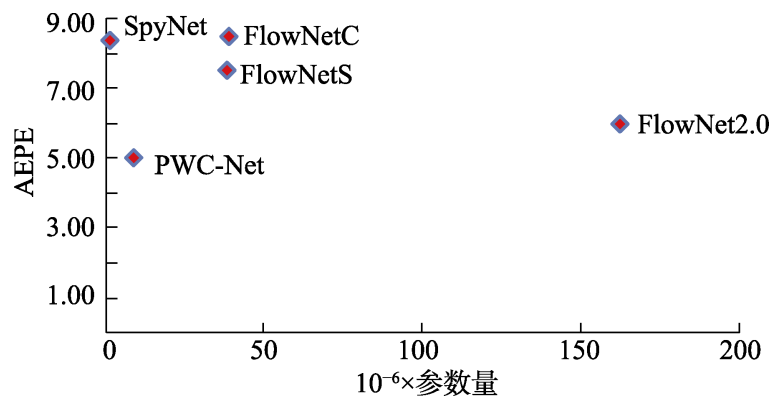

图 9 各光流估计方法的参数量-精度关系图

综上所述，目前深度光流估计的方法面临着 如下问题和挑战. 深度光流网络的泛化性能不好, 新的数据需要重新微调; 深度光流网络的训练时 间长，如何减少训练时间是下一步的研究方向; 深 度光流网络需要大量的标签, 而光流的标签很难 获得，所以如何把深度方法与传统方法充分融合 仍然是下一步研究方向; 如何在不损失精度的情 况下提高计算速度, 最终用无监督的深度网络学 习出精确的光流且达到实时性是一个挑战.

\section{4 深度光流网络与视频任务联合学习}

光流在计算机视觉中有广泛的应用，尤其是

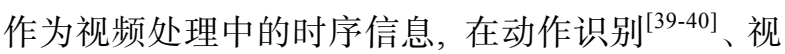
频分割 ${ }^{[41]}$ 和视频增强 ${ }^{[42]}$ 等任务中都有应用.

在深度光流网络出现之前, 光流的结果通常
作为一个任务的输人, 需要先用光流估计的方法 得到视频帧的光流，然后又作为视频任务算法的 输人，使光流与视频任务本身是 2 个分离的过程， 不能利用其相关性. 端到端网络的出现，使光流估 计和具体的任务可以通过一个深度网络联合学习共 同得到, 由任务驱动的光流估计学习出来的标准依 据最终的任务确定，最终得到的是面向任务的光流.

\section{1 深度光流估计与动作识别联合学习}

动作识别是视频分析中的重要任务之一，其 输人是一个短视频，输出是这个视频的动作标签. 光流在动作识别中通常被用作视频的时序特征, 光流在 $x$ 方向和 $y$ 方向的值所表示的灰度图作为 CNN 的输人. 视频帧的光流都是通过光流算法提前 计算好, 再作为时序特征输人到动作识别网络中.

动作识别任务并不一定需要逐像素精确光流, 而是需要与动作识别任务最相关的光流，如运动 边缘. 光流估计与动作识别联合学习就是把光流 估计和动作识别放在一个端到端的网络 ${ }^{[43-44]}$ 中进 行学习, 损失函数需要考虑 2 个任务. 如图 10 所示, 最后的损失函数包含光流和动作识别 2 部分标签.

联合学习的网络结构用的是 FlowNet1.0 中的 结构 ${ }^{[34-35]}$. 动作光流学习到的光流与传统逐像素的 精确光流不一样, 更偏向于边缘, 如图 11 学习到的 光流大部分在人体的边缘, 而不是逐像素的光流.

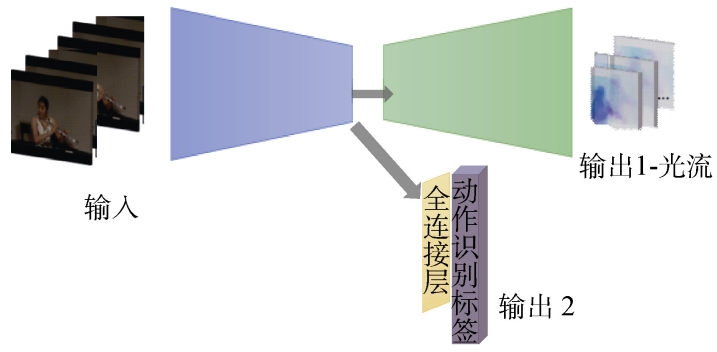

图 10 光流与动作识别联合学习网络架构 ${ }^{[43-44]}$

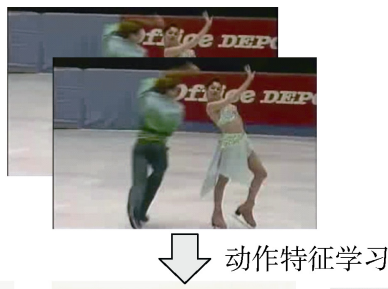

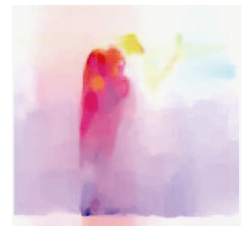

原始光流

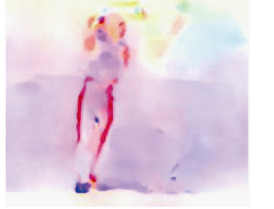

TVNet 未训练的光流

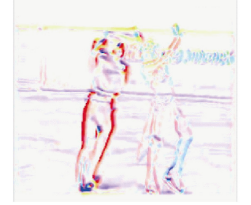

TVNet 训练的光流
图 11 原始光流与用联合网络学习到的光流 ${ }^{[45]}$ 


\section{2 深度光流估计与视频分割联合学习}

视频目标分割任务是指将输人的一段视频中 的目标分割出来, 表示方法为目标的像素为 1 , 非 目标的像素为 0 .

视频分割与光流估计任务都是逐像素的，都 需要对每个像素求值. 两者联合学习的方式是建 立 2 个分支，视频分割分支使用 $\mathrm{FCNN}$ ，光流估计 分支使用 FlowNetS 结构. 为了使 2 个分支建立起 联系，在上采样阶段设计了一个桥梁，使 2 个网络 连接起来, 使特征图可以双向传播. 损失函数的设 计结合了分割和光流 2 个任务. 该方法可以使光流 和分割任务相互学习，相互促进.

\section{3 深度光流估计与视频增强任务联合学习}

视频增强技术是指为了提高视频质量对视频 帧做一系列处理. 视频增强任务包括去噪、超分辨 率、插帧等, 输人为原始视频帧, 输出为质量更高 的视频帧.

在视频增强算法中, 光流经常作为视频帧序 列之间的时序特征. 文献[42]提出一种面向任务的 光流，把光流与视频增强的重要技术联合起来学 习. 其网络框架如图 12 所示, 输人一段视频, 先通 过光流网络学习光流, 得到的结果与参考帧进行 变形后作为视频处理网络的输人. 光流网络框架 使用 $\mathrm{SpyNet}^{[19]}$, 但是其是自学习的、没有光流标 签的.

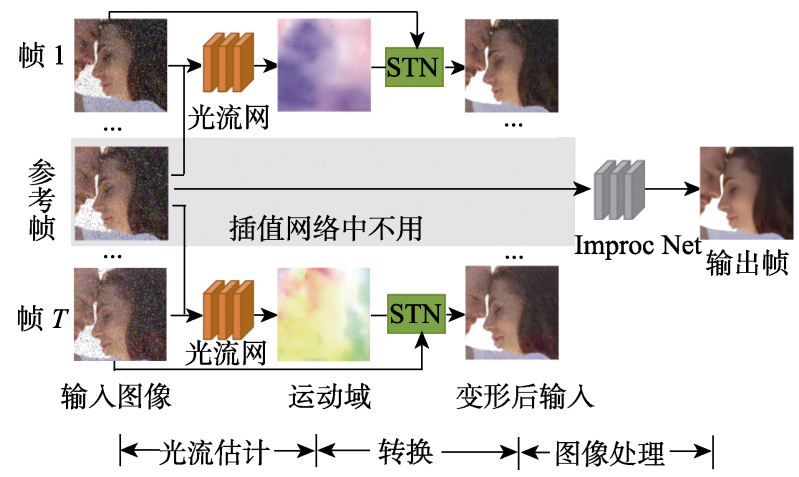

图 12 视频去噪与光流联合学习网络图 ${ }^{[42]}$

通过图 13 可以看出，由任务驱动得到的光流 值，不同任务的光流是有区别的，光流任务本身定 义的逐像素的光流值对任务并不是最优的选择.

光流在视频分析方面的应用还有视频目标检 测 ${ }^{[46-47]}$ 、姿态识别 ${ }^{[48]}$ 、视频中的衣物分割 ${ }^{[49]}$ 等. 深 度网络的出现，使光流和视频中的特定任务可以 相互学习, 相互促进, 获得由任务驱动的光流. 进

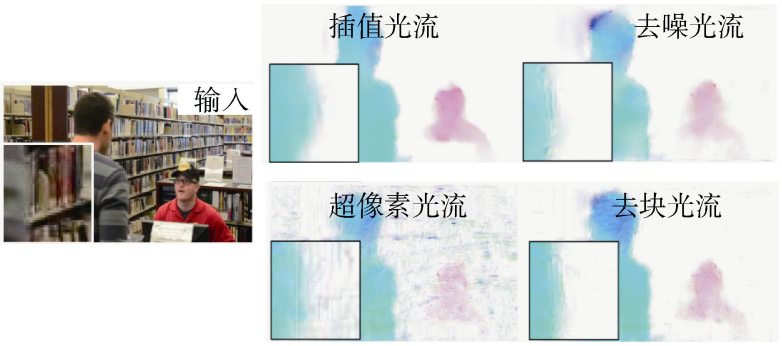

图 13 不同任务得到的不同光流 ${ }^{[42]}$

一步探索视频任务与光流的联合学习是很有应用 价值的研究方向.

\section{5 结 语}

基于深度学习的光流估计已经取得了一定的 发展，并且有利于与任务端到端的联合学习。通过 对现有方法的实验结果分析和总结, 得出目前基 于深度学习的光流网络还存在以下问题：(1) 学习 到的光流网络泛化性能差, 每计算一个新的数据 集合都要进行微调; (2) 光流网络的训练时间长, 需要几天, 而且需要大量的标签数据, 光流的标签 很难获得；(3) 光流估计与任务本身脱节, 逐像素 的光流估计对视频任务是圥余的. 针对这些问题 和挑战, 利用传统方法的思想设计出更轻便且泛 化性能更好的深度光流网络是未来的发展方向; 通过与特定视频任务联合学习去除高精度光流的 穴余，获得由任务驱动的光流是很有应用价值的 研究方向.

\section{参考文献(References):}

[1] Ma Songde, Zhang Zhengyou. Computer vision: calculation theory and algorithm basis[M]. Beijing: Science Press. 1998: 117-130 (in Chinese)

(马颂德, 张正友. 计算机视觉: 计算理论与算法基础 [M]. 北京: 科学出版社, 1998: 117-130)

[2] Prentice W, Gibson J J. The perception of the visual world[J]. The American Journal of Psychology, 1951, 64(3): 440-444

[3] Baker S, Scharstein D, Lewis J P, et al. A database and evaluation methodology for optical flow[J]. International Journal of Computer Vision, 2011, 92(1): 1-31

[4] Geiger A., Lenz P, Urtasun R. Are we ready for autonomous driving? The KITTI vision benchmark suite[C] //Proceedings of the IEEE Conference on Computer Vision and Pattern Recognition. Los Alamitos: IEEE Computer Society Press, 2012: 3354-3361

[5] Kennedy R, Taylor C J. Optical flow with geometric occlusion estimation and fusion of multiple frames[C] //Proceedings of 
the International Workshop on Energy Minimization Methods in Computer Vision and Pattern Recognition. Heidelberg: Springer, 2015: 364-377

[6] Butler D J, Wulff J, Stanley G B, et al. A naturalistic open source movie for optical flow evaluation[C] //Proceedings of the IEEE International Conference on Computer Vision. Los Alamitos: IEEE Computer Society Press, 2012: 611-625

[7] Dosovitskiy A, Fischer P, Ilg E, et al. FlowNet: learning optical flow with convolutional networks[C]//Proceedings of the IEEE International Conference on Computer Vision. Los Alamitos: IEEE Computer Society Press, 2015: 2758-2766

[8] Mayer N, Ilg E, Häusser P, et al. A large dataset to train convolutional networks for disparity, optical flow, and scene flow estimation[C] //Proceedings of the IEEE Conference on Computer Vision and Pattern Recognition. Los Alamitos: IEEE Computer Society Press, 2016: 4040-4048

[9] Janai J, Güney F, Wulff J, et al. Slow Flow: exploiting high-speed cameras for accurate and diverse optical flow reference data[C] //Proceedings of the IEEE Conference on Computer Vision and Pattern Recognition. Los Alamitos: IEEE Computer Society Press, 2017: 1406-1416

[10] Brox T, Malik J. Large displacement optical flow: descriptor matching in variational motion estimation[J]. IEEE Transactions on Pattern Analysis and Machine Intelligence, 2011, 33(3): 500-513

[11] Horn B K P, Schunck B G. Determining optical flow[J]. Artificial Intelligence, 1981, 17(1-3): 185-203

[12] Song Shuang, Yang Jian, Wang Yongtian. Technology and prospect of global optical flow[J]. Journal of Computer-Aided Design \& Computer Graphics, 2014, 26(5): 841-850(in Chinese)

(宋爽, 杨健, 王涌天. 全局光流场估计技术及展望 [J]. 计算 机辅助设计与图形学学报, 2014, 26(5): 841-850)

[13] Sun D Q, Roth S, Black M J. A quantitative analysis of current practices in optical flow estimation and the principles behind them[J]. International Journal of Computer Vision, 2014, 106(2): 115-137

[14] Black M J, Anandan P. The robust estimation of multiple motions: parametric and piecewise-smooth flow fields[J]. Computer Vision and Image Understanding, 1996, 63(1): 75-104

[15] Papenberg N, Bruhn A, Brox T, et al. Highly accurate optic flow computation with theoretically justified warping[J]. International Journal of Computer Vision, 2006, 67(2): 141-158

[16] Werlberger M, Trobin W, Pock T, et al. Anisotropic huber- $L^{1}$ optical flow[C] //Proceedings of the British Machine Vision Conference. Durham: BMVA Press, 2009: 1-11

[17] Lucas B D, Kanade T. An iterative image registration technique with an application to stereo vision[C] //Proceedings of the International Joint Conferences on Artificial Intelligence. San Mateo: Morgan Kaufmann Publishers, 1981: 674-679

[18] Bouguet J Y. Pyramidal implementation of the affine Lucas Kanade feature tracker description of the algorithm[J]. Intel Corporation, 2001, 5: 1-10

[19] Ranjan A, Black M J. Optical flow estimation using a spatial pyramid network[C] //Proceedings of the IEEE Conference on Computer Vision and Pattern Recognition. Los Alamitos: IEEE Computer Society Press, 2017: 2720-2729
[20] Ilg E, Mayer N, Saikia T, et al. FlowNet 2.0: evolution of optical flow estimation with deep networks[C] //Proceedings of the IEEE Conference on Computer Vision and Pattern Recognition. Los Alamitos: IEEE Computer Society Press, 2017: 1647-1655

[21] Sun D Q, Yang X D, Liu M Y, et al. PWC-Net: CNNs for optical flow using pyramid, warping, and cost volume[C] // Proceedings of the IEEE Conference on Computer Vision and Pattern Recognition. Los Alamitos: IEEE Computer Society Press, 2018: 8934-8943

[22] Hui T W, Tang X O, Loy C C. LiteFlowNet: a lightweight convolutional neural network for optical flow estimation[C] //Proceedings of the IEEE Conference on Computer Vision and Pattern Recognition. Los Alamitos: IEEE Computer Society Press, 2018: 8981-8989

[23] Hur J, Roth S. Iterative residual refinement for joint optical flow and occlusion estimation[OL]. [2019-07-02]. https://arxiv. org/abs/1904.09117? context=cs

[24] Huang G, Liu Z, Maaten L V D, et al. Densely connected convolutional networks[C] //Proceedings of the IEEE Conference on Computer Vision and Pattern Recognition. Los Alamitos: IEEE Computer Society Press, 2017: 2261-2269

[25] Yu F, Koltun V. Multi-scale context aggregation by dilated convolutions[OL]. [2019-07-02]. https://arxiv.org/abs/1511. 07122

[26] Yin Z C, Darrell T, Yu F. Hierarchical discrete distribution decomposition for match density estimation[OL]. [2019-07-02]. https://arxiv.org/abs/1812.06264

[27] Ilg E, Çiçek Ö, Galesso S, et al. Uncertainty estimates and multi-hypotheses networks for optical flow[C] //Proceedings of the European Conference on Computer Vision. Heidelberg: Springer, 2018: 677-693

[28] Ren Z L, Gallo O, Sun D Q, et al. A fusion approach for multi-frame optical flow estimation[C] //Proceedings of the IEEE Winter Conference on Applications of Computer Vision. Los Alamitos: IEEE Computer Society Press, 2019: 2077-2086

[29] Yu J J, Harley A W, Derpanis K G. Back to basics: unsupervised learning of optical flow via brightness constancy and motion smoothness[C] //Proceedings of the European Conference on Computer Vision Workshops. Heidelberg: Springer, 2016: 3-10

[30] Liu P P, Lyu M, King I, et al. SelFlow: self-supervised learning of optical flow[OL]. [2019-07-02]. https://arxiv.org/abs/1904. 09117

[31] Lai W S, Huang J B, Yang M H. Semi-supervised learning for optical flow with generative adversarial networks[C] // Proceedings of the Advances in Neural Information Processing Systems. San Mateo: Morgan Kaufmann Publishers, 2017: 354-364

[32] Gadot D, Wolf L. PatchBatch: a batch augmented loss for optical flow $[\mathrm{C}] / /$ Proceedings of the IEEE Conference on Computer Vision and Pattern Recognition. Los Alamitos: IEEE Computer Society Press, 2016: 4236-4245

[33] Revaud J, Weinzaepfel P, Harchaoui Z, et al. EpicFlow: edge-preserving interpolation of correspondences for optical flow[C] //Proceedings of the IEEE Conference on Computer Vision and Pattern Recognition. Los Alamitos: IEEE Computer Society Press, 2015: 1164-1172

[34] Bailer C, Taetz B, Stricker D. Flow fields: dense correspon- 
dence fields for highly accurate large displacement optical flow estimation[C] // Proceedings of the IEEE Conference on International Conference on Computer Vision. Los Alamitos: IEEE Computer Society Press, 2015: 4015-4023

[35] Chen Q F, Koltun V. Full flow: optical flow estimation by global optimization over regular grids[C] //Proceedings of the IEEE Conference on Computer Vision and Pattern Recognition. Los Alamitos: IEEE Computer Society Press, 2016: 4706-4714

[36] Wulff J, Sevilla-Lara L, Black M J. Optical flow in mostly rigid scenes[C] //Proceedings of the IEEE Conference on Computer Vision and Pattern Recognition. Los Alamitos: IEEE Computer Society Press, 2017: 6911-6920

[37] Barron J L, Fleet D J, Beauchemin S S, et al. Performance of optical flow techniques[J]. International Journal of Computer Vision, 1994, 12(1): 43-77

[38] Xu J, Ranftl R, Koltun V. Accurate optical flow via direct cost volume processing[C] //Proceedings of the IEEE Conference on Computer Vision and Pattern Recognition. Los Alamitos: IEEE Computer Society Press, 2017: 5807-5815

[39] Simonyan K, Zisserman A. Two-stream convolutional networks for action recognition in videos[C] //Proceedings of the Advances in Neural Information Processing Systems. San Mateo: Morgan Kaufmann Publishers, 2014: 568-576

[40] Feichtenhofer C, Pinz A, Wildes R P. Spatiotemporal residual networks for video action recognition[C] //Proceedings of the Advances in Neural Information Processing Systems. San Mateo: Morgan Kaufmann Publishers, 2016: 3468-3476

[41] Cheng J C, Tsai Y H, Wang S J, et al. SegFlow: joint learning for video object segmentation and optical flow[C] // Proceedings of the IEEE International Conference on Computer Vision. Los Alamitos: IEEE Computer Society Press, 2017: 686-695
[42] Xue T F, Chen B A, Wu J J, et al. Video enhancement with task-oriented flow[J]. International Journal of Computer Vision, 2019, 127(8): 1106-1125

[43] Ng J Y H, Choi J, Neumann J, et al. ActionFlowNet: learning motion representation for action recognition[C] //Proceedings of the IEEE Conference on Winter Conference on Applications of Computer Vision. Los Alamitos: IEEE Computer Society Press, 2018: 1616-1624

[44] Zhu Y, Lan Z Z, Newsam S, et al. Hidden two-stream convolutional networks for action recognition[C] //Proceedings of Asian Conference on Computer Vision. Heidelberg: Springer, 2018: 363-378

[45] Fan L J, Huang W B, Gan C, et al. End-to-end learning of motion representation for video understanding $[\mathrm{C}] / /$ Proceedings of the IEEE Conference on Computer Vision and Pattern Recognition. Los Alamitos: IEEE Computer Society Press, 2018: 6016-6025

[46] Zhu X Z, Wang Y J, Dai J F, et al. Flow-guided feature aggregation for video object detection[C] //Proceedings of the IEEE Conference on International Conference on Computer Vision. Los Alamitos: IEEE Computer Society Press, 2017: 408-417

[47] Zhu X Z, Xiong Y W, Dai J F, et al. Deep feature flow for video recognition $[\mathrm{C}] / /$ Proceedings of the IEEE Conference on Computer Vision and Pattern Recognition. Los Alamitos: IEEE Computer Society Press, 2017: 4141-4150

[48] Xiu Y L, Li J F, Wang H Y, et al. Pose flow: efficient online pose tracking[OL]. [2019-07-02]. https://arxiv.org/abs/1802. 00977

[49] Liu S, Wang C H, Qian R H, et al. Surveillance video parsing with single frame supervision[C] //Proceedings of the IEEE Conference on Computer Vision and Pattern Recognition. Los Alamitos: IEEE Computer Society Press, 2017: 1013-102 